\title{
COMBINING ABILITY AND HETEROSIS EFFECTS IN SUNFLOWER OF BYELORUSSIAN ORIGIN
}

\author{
Volotovich, A.A. , Silkova, T.A., Fomchenko, N.S., Prokhorenko, O.V., \\ Davydenko, O.G.
}

Laboratory of Extrachromosomal Inheritance, Institute of Genetics and Cytology of National Academy of Sciences, Minsk 220 000, Republic of Belarus

Received: December 20, 2006

Accepted: January 10, 2008

\section{SUMMARY}

Twenty-eight hybrids were generated by crossing seven male sterile lines with four restorers in the line $\times$ tester cross system. GCA and SCA effects of parental lines and heterosis effect of $\mathrm{F}_{1}$ hybrids have been evaluated for 4 characters. Two of the twenty-eight Byelorussian hybrids (Donskoy 22 and Signal) revealed higher values for oil yield with respect to standard checks. According to the data obtained it is quite possible to produce prospective Byelorussian hybrids under local soil-climatic conditions.

Key words: GCA effects, SCA effects, line $\times$ tester analysis, heterosis

\section{INTRODUCTION}

Sharp global climate changes, intensive development of market economy, increase in industrial production, along with improvement of scientific methods and approaches create the necessary prerequisites for introduction of nontraditional plant species.

Edible oil industry has been organized in the Republic of Belarus. Oil-type sunflower (Helianthus annuus L.), as a source of high-quality vegetable oil for food industry and protein-rich oil cake and solvent cake for cattle feed, is a new crop in Belarus.

The climate of Belarus is humid, with moderate temperatures in spring and summer. Preliminary ecological trials of different sunflower varieties and hybrids of foreign origin conducted under climate conditions of Belarus were successful and indicated possibilities to grow sunflowers on an industrial scale on the territory of Belarus (Silkova et al., 2000).

The ecological trial of ten single-cross interline sunflower hybrids (bred in Rostov-on-Don) was carried out for three years (1999-2001) under local conditions, to

* Corresponding author: Phone: (+375) 17 280-82-00; Fax: (+375) 17 263-58-27; e-mail: pixies@tut.by 
assess the variability in $\mathrm{F}_{1}$ hybrid productivity parameters depending on genotypic and environmental factors. The year factor was shown to make a major contribution to the variability in seed weight per head, plant height, head diameter, 1000seed weight, oil content and percent of healthy plants before harvest. Genotype of the female plant in hybrids has a significant effect on the value of all agronomic traits tested, including seed hull content (Silkova et al., 2002).

At present, there are fifteen sunflower hybrids of foreign origin in the National Variety Register of Belarus, including the hybrid Donskoy 962 developed in collaboration with breeders from Don Branch of the All-Union Research Institute of Oil Crops (AURIOC) (Starovoitov, 2005).

In 1998, research work was initiated at the laboratory for extrachromosomal inheritance at the Institute of Genetics and Cytology of National Academy of Sciences of Belarus. It was aimed at the development of single-cross interline sunflower hybrids based on CMS, adapted to local soil-climatic conditions (Davydenko et al., 2002; 2003). Investigations were conducted in collaboration with breeders of L. A. Zhdanov Don Branch of the AURIOC (Rostov-on-Don, Russia) under the support of Soya-North Co., Ltd.

Combining ability estimation for basic productivity traits (oil content, seed- and oil yield per unit area) in parent inbred lines is necessary for selecting promising sunflower hybrids. The objectives of this study were (i) to assess the importance of GCA and SCA effects of some inbred lines in the inheritance of productivity in $\mathrm{F}_{1}$ hybrids and (ii) to estimate the degree of heterosis over a better parent and over standard checks for different productivity traits in sunflower hybrids of Byelorussian origin.

\section{MATERIALS AND METHODS}

An investigation was carried out in 2004 at the Biological Experiment Station of the Institute of Genetics and Cytology, Minsk (latitude 53 $58^{\prime} \mathrm{N}$, longitude $27^{\circ} 41^{\prime} \mathrm{E}$ ). Soil type was sod-podzolic, light loam, with a neutral reaction. Twenty-eight hybrids generated by crossing seven male sterile lines M151/04A, M152/04A, M153/04A, M154/04A, M156/04A, M157/04A, M158/04A $\left(\mathrm{BC}_{3}, \mathrm{BC}_{4}\right)$ with four restorers M178/ 04, M179/04, M180/04, M181/04 ( $\left.\mathrm{I}_{5}\right)$ were evaluated.

All crosses were assessed in replicated ( 3 plots/cross) and randomized singlerow plots (40 plants/plot). Plant spacing was $60 \mathrm{~cm}$ between rows and $60 \mathrm{~cm}$ between plants in the row (corresponding to 28,000 plant/ha). Two-way analysis of variance was used to partition the general combining ability (GCA) of the parent lines and the specific combining ability (SCA) of the progenies. The analysis of variance was calculated through GRIF 1 module of AB-Stat 2.1 developed at the Institute of Genetics and Cytology, Minsk. The computation of GCA and SCA effects was done by the line $\times$ tester analysis technique of Kempthorne (1957). The components of genotypic variance were calculated according to Plokhynskiy (1964). The 
traits analyzed were seed weight per head, head diameter, oil and hull content of seed. The oil content data were obtained by diethyl ether extraction technique published in GOST 13979.2-94 BY. The degree of heterosis over better parent and over standard check was estimated according to Fonesca and Patterson (1968).

\section{RESULTS AND DISCUSSION}

The one-way analysis of variance revealed significant differences between the $F_{1}$ hybrids for all parameters under study.

The two-way analysis of combining ability variance, summarized in Table 1, provided evidence of highly significant levels of GCA and SCA variances for most characters.

Table 1: Two-way analysis of combining ability in sunflower

\begin{tabular}{lccccc}
\hline \multirow{2}{*}{ Source of variation } & \multirow{2}{*}{ Df } & \multicolumn{4}{c}{ Mean square } \\
\cline { 3 - 6 } & & Seed weight per head & Head diameter & Oil content & Hull content \\
\hline GCA of CMS lines & 6 & $619.56^{\star *}$ & $13.12^{\star *}$ & $4.68^{\star *}$ & $10.30^{\star *}$ \\
GCA of restorers & 3 & $222.97^{\star *}$ & $5.38^{*}$ & $11.98^{\star *}$ & $1.85^{\star *}$ \\
SCA & 18 & $85.81^{* *}$ & 2.60 & $3.43^{\star *}$ & $1.20^{\star *}$ \\
Random deviation & 54 & 37.82 & 1.71 & 0.02 & 0.42 \\
& \multicolumn{7}{c}{ Component of genotypic variance } \\
$\sigma^{2}$ GCA of CMS lines & 133.44 & 2.63 & 0.31 & 2.28 \\
$\sigma^{2}$ GCA of restorers & 19.59 & 0.40 & 1.22 & 0.09 \\
$\sigma^{2}$ SCA & 47.99 & 0.89 & 3.41 & 0.78 \\
$\sigma^{2}$ GCA of CMS lines $/ \sigma^{2}$ SCA & 2.78 & 2.96 & 0.09 & 2.92 \\
$\sigma^{2}$ GCA of restorers $/ \sigma^{2}$ SCA & 0.41 & 0.45 & 0.36 & 0.12 \\
\hline
\end{tabular}

Significance levels: ${ }^{*} 0.01<P \leq 0.05 ;{ }^{* \star} P \leq 0.01$

Table 2: General combining ability effects in $c m s$ lines and restorers

\begin{tabular}{lcccc}
\hline Line & \multicolumn{4}{c}{ Character } \\
\cline { 2 - 5 } & Seed weight per head & Head diameter & Oil content & Hull content \\
\hline M151/04A $\left(\mathrm{BC}_{4}\right)$ & 19.00 & 1.53 & -0.32 & 1.86 \\
M152/04A $\left(\mathrm{BC}_{3}\right)$ & -1.41 & 0.13 & 2.04 & -2.55 \\
M153/04A $\left(\mathrm{BC}_{3}\right)$ & -10.24 & -0.92 & 0.68 & -1.56 \\
M154/04A $\left(\mathrm{BC}_{3}\right)$ & -10.48 & -2.98 & -0.21 & -0.46 \\
M156/04A $\left(\mathrm{BC}_{3}\right)$ & 16.20 & 2.71 & -0.07 & 1.32 \\
M157/04A $\left(\mathrm{BC}_{3}\right)$ & -7.99 & 0.05 & -0.46 & 0.43 \\
M158/04A $\left(\mathrm{BC}_{3}\right)$ & -5.08 & -0.52 & -0.65 & 0.95 \\
\hline Standard error & 18.91 & 0.85 & 0.01 & 0.21 \\
\hline M178/04Rf $\left(I_{5}\right)$ & 4.93 & 1.21 & -1.05 & -0.45 \\
M179/04Rf $\left(I_{5}\right)$ & -2.32 & -0.55 & -0.98 & -0.44 \\
M180/04Rf $\left(I_{5}\right)$ & -6.90 & 0.08 & 0.29 & 0.49 \\
M181/04Rf $\left(I_{5}\right)$ & 4.29 & -0.74 & 1.73 & 0.40 \\
\hline Standard error & 10.80 & 0.49 & 0.01 & 0.12 \\
\hline
\end{tabular}


The relative genetic control of characters may be judged by the ratio of GCA and SCA variances which indicated that in the female lines, seed weight per head, head diameter and hull content were predominantly controlled by additive gene effects and oil content by dominant and epistatic ones (Table 1). The data obtained confirm the earlier established fact that the oil content is mainly determined by dominant and epistatic gene effects (Kovacik and Skaloud, 1972; Volotovich et al., 2005).

GCA effects of the lines under study are presented in Table 2. Two female lines (M151/04A and M156/04A) were revealed to have a high positive GCA effect for seed weight per head, which is one of the basic productivity parameters. The lines M152/ 04A and M153/04A demonstrated a positive GCA effect for seed oil content.

Table 3: Specific combining ability effects $\left(\mathrm{S}_{\mathrm{ij}}\right)$ and variances $\left(\sigma^{2} \mathrm{Si}\right)$ of cross combinations

\begin{tabular}{|c|c|c|c|c|c|c|c|c|}
\hline \multirow[t]{3}{*}{ Cross } & \multicolumn{8}{|c|}{ Character } \\
\hline & \multicolumn{2}{|c|}{ Seed weight per head } & \multicolumn{2}{|c|}{ Head diameter } & \multicolumn{2}{|c|}{ Oil content } & \multicolumn{2}{|c|}{ Hull content } \\
\hline & $\mathrm{S}_{\mathrm{ij}}$ & $\sigma^{2} \mathrm{Si}$ & $\mathrm{S}_{\mathrm{ij}}$ & $\sigma^{2} \mathrm{Si}$ & $\mathrm{S}_{\mathrm{ij}}$ & $\sigma^{2} \mathrm{Si}$ & $\mathrm{S}_{\mathrm{ij}}$ & $\sigma^{2} \mathrm{Si}$ \\
\hline $\mathrm{M} 151 / 04 \mathrm{~A} \times \mathrm{M} 178 / 04 \mathrm{Rf}$ & 11.68 & 45.55 & -0.35 & -0.90 & 1.48 & 1.50 & 0.06 & 0.67 \\
\hline $\mathrm{M} 151 / 04 \mathrm{~A} \times \times \mathrm{M} 179 / 04 \mathrm{Rf}$ & -0.78 & & 0.65 & & -0.29 & & -0.35 & \\
\hline M151/04A×M180/04Rf & -2.90 & & -0.23 & & -1.47 & & 1.29 & \\
\hline M151/04A×M181/04Rf & -8.01 & & -0.07 & & 0.28 & & -1.00 & \\
\hline M152/04A×M178/04Rf & -8.65 & 222.77 & -0.79 & 0.59 & 0.21 & 3.53 & -0.69 & -0.04 \\
\hline M152/04A×M179/04Rf & 9.59 & & -1.41 & & 2.51 & & 0.33 & \\
\hline $\mathrm{M} 152 / 04 \mathrm{~A} \times \mathrm{M} 180 / 04 \mathrm{Rf}$ & 16.47 & & 1.16 & & -1.85 & & 0.29 & \\
\hline M152/04A×M181/04Rf & -17.41 & & 1.04 & & -0.87 & & 0.07 & \\
\hline $\mathrm{M} 153 / 04 \mathrm{~A} \times \mathrm{M} 178 / 04 \mathrm{Rf}$ & -5.79 & 19.08 & -0.95 & 0.80 & -0.63 & 0.63 & -1.47 & 1.03 \\
\hline M153/04A×M179/04Rf & -4.01 & & -0.31 & & -0.71 & & 1.24 & \\
\hline M153/04A $\times M 180 / 04 R f$ & 8.94 & & 2.02 & & 0.89 & & -0.17 & \\
\hline M153/04A $\times M 181 / 04 R f$ & 0.86 & & -0.76 & & 0.45 & & 0.40 & \\
\hline M154/04A×M178/04Rf & -1.98 & -16.71 & 0.58 & 4.24 & -2.18 & 2.60 & 2.05 & 2.06 \\
\hline M154/04A $\times M 179 / 04 R f$ & 0.00 & & 1.25 & & 1.69 & & -1.58 & \\
\hline M154/04A×M180/04Rf & -1.92 & & 1.57 & & 0.47 & & 0.08 & \\
\hline M154/04A $\times$ M181/04Rf & 3.90 & & -3.41 & & 0.02 & & -0.55 & \\
\hline M156/04A×M178/04Rf & 0.01 & 31.55 & 1.77 & 1.66 & 0.30 & 0.48 & 0.05 & -0.17 \\
\hline M156/04A $\times$ M179/04Rf & 4.12 & & -0.28 & & -0.09 & & 0.03 & \\
\hline M156/04A $\times M 180 / 04 R f$ & -10.50 & & -2.15 & & 0.71 & & -0.42 & \\
\hline M156/04A $\times$ M181/04Rf & 6.36 & & 0.65 & & -0.92 & & 0.34 & \\
\hline $\mathrm{M} 157 / 04 \mathrm{~A} \times \mathrm{M} 178 / 04 \mathrm{Rf}$ & -1.80 & -3.59 & -0.16 & -0.66 & 2.29 & 10.18 & -0.44 & 0.15 \\
\hline M157/04A $\times M 179 / 04 R f$ & -0.49 & & 0.88 & & -4.71 & & -0.10 & \\
\hline M157/04A $\times M 180 / 04 R f$ & -4.15 & & -0.71 & & 0.99 & & 0.94 & \\
\hline M157/04A $\times$ M181/04Rf & 6.44 & & -0.01 & & 1.43 & & -0.40 & \\
\hline M158/04A $\times M 178 / 04 R f$ & 6.52 & 46.02 & -0.10 & 2.23 & -1.47 & 1.66 & 0.44 & 1.65 \\
\hline M158/04A $\times$ M179/04Rf & -8.44 & & -0.78 & & 1.61 & & 0.43 & \\
\hline M158/04A $\times M 180 / 04 R f$ & -5.95 & & -1.67 & & 0.25 & & -2.02 & \\
\hline M158/04A×M181/04Rf & 7.87 & & 2.56 & & -0.39 & & 1.15 & \\
\hline Standard error & & & & & & & & \\
\hline
\end{tabular}


Table 4: Heterosis effects for different traits

\begin{tabular}{|c|c|c|c|c|c|c|c|c|}
\hline \multirow{3}{*}{$\begin{array}{l}\text { Cross combination, } \\
\text { or female line }\end{array}$} & \multicolumn{8}{|c|}{ Character } \\
\hline & \multicolumn{2}{|c|}{$\begin{array}{l}\text { Seed weight } \\
\text { per head }\end{array}$} & \multicolumn{2}{|c|}{ Head diameter } & \multicolumn{2}{|c|}{ Oil content } & \multicolumn{2}{|c|}{ Hull content } \\
\hline & $X, \mathrm{~g}$ & $H_{\mathrm{F} 1}, \%$ & $X, \mathrm{~cm}$ & $H_{\mathrm{F} 1}, \%$ & $X, \%$ & $H_{\mathrm{F} 1}, \%$ & $X, \%$ & $H_{\mathrm{F} 1}, \%$ \\
\hline $\mathrm{M} 151 / 04\left(I_{5}\right)$ & 25.17 & - & 13.52 & - & 39.47 & - & 24.97 & - \\
\hline M151/04A $\times M 178 / 04 R f$ & 113.80 & $352.13^{* *}$ & 22.37 & $65.46^{* *}$ & 45.51 & $15.30^{\star *}$ & 26.40 & 5.73 \\
\hline M151/04A $\times M 179 / 04 R f$ & 94.10 & $273.86^{* *}$ & 21.90 & 54.59 ** & 43.80 & $10.97^{\star \star}$ & 26.00 & 4.12 \\
\hline $\mathrm{M} 151 / 04 \mathrm{~A} \times \mathrm{M} 180 / 04 \mathrm{Rf}$ & 87.40 & $247.24^{\star *}$ & 21.38 & $58.14 * \star$ & 43.90 & $11.22^{\star \star}$ & 28.57 & $14.42^{\star \star}$ \\
\hline M151/04A $\times M 181 / 04 R f$ & 93.47 & $271.35^{\star \star}$ & 20.66 & $52.81^{* *}$ & 47.08 & $19.28^{\star \star}$ & 26.18 & 4.85 \\
\hline $\mathrm{M} 152 / 04\left(\mathrm{I}_{5}\right)$ & 35.33 & - & 16.25 & - & 40.86 & - & 21.77 & - \\
\hline M152/04A $\times M 178 / 04 R f$ & 73.07 & $106.82^{\star *}$ & 20.70 & $27.38^{\star \star}$ & 47.59 & $16.47^{\star \star}$ & 21.23 & -2.48 \\
\hline M152/04A×M179/04Rf & 84.07 & $137.96^{\star \star}$ & 19.09 & $17.48^{*}$ & 49.97 & $22.30^{\star *}$ & 22.27 & 2.30 \\
\hline M152/04A $\times M 180 / 04 R f$ & 86.37 & $144.47^{\star}$ & 21.37 & $31.51^{* *}$ & 46.87 & $14.71^{* *}$ & 23.15 & 6.34 \\
\hline M152/04A×M181/04Rf & 63.67 & $80.22^{*}$ & 20.43 & $25.72^{\star \star}$ & 49.29 & $20.63^{\star \star}$ & 22.85 & 4.96 \\
\hline$M 153 / 04\left(I_{5}\right)$ & 26.00 & - & 12.18 & - & 41.04 & - & 26.03 & - \\
\hline M153/04A $\times M 178 / 04 R f$ & 67.10 & $158.05^{\star \star}$ & 19.34 & $58.78^{\star \star}$ & 45.40 & $10.62^{\star \star}$ & 21.45 & $-17.60 * *$ \\
\hline M153/04A $\times M 179 / 04 R f$ & 61.63 & $137.04^{* *}$ & 18.60 & $52.71^{\star *}$ & 45.38 & $10.58^{\star \star}$ & 24.17 & -7.15 \\
\hline M153/04A $\times M 180 / 04 R f$ & 70.67 & $171.81^{* *}$ & 21.18 & $73.89^{\star *}$ & 48.25 & $17.57^{\star \star}$ & 23.68 & $-9.03^{*}$ \\
\hline $\mathrm{M} 153 / 04 \mathrm{~A} \times \mathrm{M} 181 / 04 \mathrm{Rf}$ & 73.10 & $181.15^{\star \star}$ & 17.71 & 45.40 ** & 49.25 & $20.00^{\star \star}$ & 24.17 & -7.15 \\
\hline$M 154 / 04\left(I_{5}\right)$ & 26.00 & - & 16.25 & - & 39.60 & - & 24.70 & - \\
\hline M154/04A $\times$ M178/04Rf & 70.67 & $171.81^{\star *}$ & 18.89 & $16.25^{\star \star}$ & 42.96 & $8.48^{* \star}$ & 26.07 & 5.55 \\
\hline M154/04A $\times$ M179/04Rf & 65.43 & $151.65^{\star *}$ & 17.83 & $9.72^{\star}$ & 46.89 & $18.41^{\text {** }}$ & 22.45 & -9.11 \\
\hline M154/04A $\times M 180 / 04 R f$ & 58.90 & $126.54^{\star *}$ & 18.67 & $14.89^{\star}$ & 46.95 & $18.56^{\star \star}$ & 25.03 & 1.34 \\
\hline M154/04A $\times$ M181/04Rf & 75.90 & $191.92^{\star *}$ & 19.08 & $17.42^{\star \star}$ & 47.93 & $21.04^{\star \star}$ & 24.32 & -1.54 \\
\hline$M 156 / 04\left(I_{5}\right)$ & 34.43 & - & 22.67 & - & 32.88 & - & 30.30 & - \\
\hline M156/04A $\times M 178 / 04 R f$ & 100.67 & $192.39 * *$ & 25.28 & $11.51^{*}$ & 45.58 & $38.63^{* *}$ & 25.85 & $-14.69 * *$ \\
\hline M156/04A $\times$ M179/04Rf & 96.20 & $180.22^{\star *}$ & 21.88 & -3.48 & 45.25 & $37.62^{\star *}$ & 25.83 & $-14.75^{\star \star}$ \\
\hline M156/04A $\times$ M180/04Rf & 77.00 & 123.64 ** & 20.63 & $-9.00^{*}$ & 47.33 & $43.95^{\star \star}$ & 26.32 & $-13.14 * *$ \\
\hline M156/04A $\times M 181 / 04 R f$ & 105.03 & $205.05^{\star *}$ & 22.71 & 0.18 & 47.14 & $43.37^{\star \star}$ & 26.98 & $-10.96 * *$ \\
\hline $\mathrm{M} 157 / 04\left(\mathrm{I}_{5}\right)$ & 36.66 & - & 15.32 & - & 40.15 & - & 27.47 & - \\
\hline M157/04A $\times$ M178/04Rf & 73.33 & $100.03^{\star}$ & 20.96 & 36.81 ** & 47.18 & $17.51^{\text {** }}$ & 24.47 & $-10.92^{*}$ \\
\hline M157/04A $\times M 179 / 04 R f$ & 67.40 & 83.85 & 20.38 & $33.03^{* *}$ & 40.24 & 0.22 & 24.82 & -9.65 \\
\hline M157/04A $\times$ M180/04Rf & 59.17 & 61.40 & 19.42 & $26.76^{\star *}$ & 47.23 & $17.63^{\star *}$ & 26.78 & -2.51 \\
\hline M157/04A $\times M 181 / 04 R f$ & 80.93 & $120.76^{\star \star}$ & 19.22 & $25.46^{\star *}$ & 49.10 & $22.29^{\star *}$ & 25.35 & -7.72 \\
\hline$M 158 / 04\left(I_{5}\right)$ & 24.57 & - & 13.30 & - & 36.91 & - & 31.75 & - \\
\hline M158/04A $\times$ M178/04Rf & 84.57 & $244.20 *$ & 20.58 & $54.74^{\star *}$ & 43.23 & $17.12^{\star \star}$ & 25.87 & $-18.52^{* *}$ \\
\hline M158/04A $\times M 179 / 04 R f$ & 62.37 & $153.85^{\star}$ & 18.15 & $36.47^{\star \star}$ & 46.38 & $25.66^{\star \star}$ & 25.87 & $-18.52^{\star \star}$ \\
\hline M158/04A $\times M 180 / 04 R f$ & 60.27 & 145.30 ** & 17.88 & $34.44^{\star *}$ & 46.29 & $25.41^{* \star}$ & 24.35 & $-23.31 * *$ \\
\hline M158/04A×M181/04Rf & 85.27 & $247.05^{\star \star}$ & 21.24 & $59.70^{\star \star}$ & 47.09 & $27.58^{\star \star}$ & 27.43 & $-13.61^{* *}$ \\
\hline
\end{tabular}

Significance levels: * $0.01<P \leq 0.05 ;{ }^{* *} P \leq 0.01$ 
Four inbred lines, selected in terms of high GCA, exhibited a high positive SCA variance for seed weight per head and a low positive SCA variance for oil content in seed (Table 3). The line M152/04A showed a low negative GCA effect and a high positive SCA variance for seed weight per head. This indicates that this line, if used in a certain combination, may produce high-yielding hybrids.

The SCA analysis for seed weight per head revealed 10 best cross combinations. Four combinations were best regarding oil content in seed (Table 3).

Heterosis effects of the Byelorussian hybrids are shown in Table 4. Most of the hybrids showed significant positive heterosis values for seed weight per head and oil content (Table 4). The heterosis values ranged from 61.40 to $352.13 \%$ and from 0.22 to $43.95 \%$, respectively. Twenty-five hybrids exhibited positive heterosis for head diameter. Heterosis was frequently negative for hull content.

Table 5: Heterosis over standard checks for seed yield and oil yield (metric cent/ha)

\begin{tabular}{|c|c|c|c|c|c|c|}
\hline \multirow{2}{*}{ Cross combination } & \multicolumn{3}{|c|}{ Seed yield } & \multicolumn{3}{|c|}{ Oil yield } \\
\hline & $X, \mathrm{mc} \mathrm{ha}^{-1}$ & $H_{\mathrm{SC} 1}, \%$ & $\mathrm{H}_{\mathrm{SC} 2}, \%$ & $X, \mathrm{mc} \mathrm{ha}^{-1}$ & $H_{\mathrm{SC} 1}, \%$ & $H_{\mathrm{SC} 2}, \%$ \\
\hline $\mathrm{M} 151 / 04 \mathrm{~A} \times \mathrm{M} 178 / 04 \mathrm{Rf}$ & 29.5 & 65.7 & 27.2 & 12.6 & 61.5 & 16.7 \\
\hline M151/04A $\times M 179 / 04 R f$ & 22.6 & 27.0 & -2.6 & 9.3 & 19.2 & -13.9 \\
\hline M151/04A $\times$ M180/04Rf & 24.0 & 34.8 & 3.4 & 9.9 & 26.9 & -8.3 \\
\hline M151/04A $\times$ M181/04Rf & 23.3 & 30.3 & 0.4 & 10.3 & 32.1 & -4.6 \\
\hline M152/04A $\times M 178 / 04 R f$ & 19.0 & 6.7 & -18.1 & 8.5 & 8.9 & -21.3 \\
\hline M152/04A $\times$ M179/04Rf & 22.0 & 23.6 & -5.2 & 10.3 & 32.1 & -4.6 \\
\hline $\mathrm{M} 152 / 04 \mathrm{~A} \times \mathrm{M} 180 / 04 \mathrm{Rf}$ & 21.3 & 19.7 & -8.2 & 9.4 & 20.5 & -12.9 \\
\hline $\mathrm{M} 152 / 04 \mathrm{~A} \times \mathrm{M} 181 / 04 \mathrm{Rf}$ & 18.1 & 1.7 & -22.0 & 8.4 & 7.7 & -22.2 \\
\hline M153/04A $\times$ M178/04Rf & 19.4 & 9.0 & -16.4 & 8.3 & 6.4 & -23.1 \\
\hline M153/04A $\times$ M179/04Rf & 16.2 & -9.0 & -30.2 & 6.9 & -11.5 & -36.1 \\
\hline M153/04A $\times$ M180/04Rf & 20.3 & 14.0 & -12.5 & 9.2 & 17.9 & -14.8 \\
\hline $\mathrm{M} 153 / 04 \mathrm{~A} \times \mathrm{M} 181 / 04 \mathrm{Rf}$ & 19.7 & 10.7 & -15.1 & 9.1 & 16.7 & -15.7 \\
\hline M154/04A $\times$ M178/04Rf & 19.1 & 7.3 & -17.7 & 7.7 & -1.3 & -28.7 \\
\hline M154/04A $\times$ M179/04Rf & 18.2 & 2.2 & -21.6 & 8.0 & 2.6 & -25.9 \\
\hline M154/04A $\times$ M180/04Rf & 15.0 & -15.7 & -35.3 & 6.6 & -15.4 & -38.9 \\
\hline M154/04A $\times$ M181/04Rf & 21.1 & 18.5 & -9.1 & 9.5 & 21.8 & -12.0 \\
\hline M156/04A $\times$ M178/04Rf & 29.6 & 66.3 & 27.6 & 12.7 & 62.8 & 17.6 \\
\hline M156/04A $\times$ M179/04Rf & 25.2 & 41.6 & 8.6 & 10.7 & 37.2 & -0.9 \\
\hline M156/04A $\times$ M180/04Rf & 21.6 & 21.3 & -6.9 & 9.6 & 23.1 & -11.1 \\
\hline M156/04A $\times$ M181/04Rf & 19.4 & 9.0 & -16.4 & 8.6 & 10.3 & -20.4 \\
\hline M157/04A ×M178/04Rf & 17.8 & 0.0 & -23.3 & 7.9 & 1.3 & -26.9 \\
\hline M157/04A $\times$ M179/04Rf & 12.2 & -31.5 & -47.4 & 4.6 & -41.0 & -57.4 \\
\hline M157/04A $\times$ M180/04Rf & 15.5 & $-12 .-$ & -33.2 & 6.9 & -11.5 & -36.1 \\
\hline M157/04A $\times$ M181/04Rf & 21.0 & 18.0 & -9.5 & 9.7 & 24.4 & -10.2 \\
\hline $\mathrm{M} 158 / 04 \mathrm{~A} \times \mathrm{M} 178 / 04 \mathrm{Rf}$ & 16.0 & -10.1 & -31.0 & 6.5 & -16.7 & -39.8 \\
\hline M158/04A×M179/04Rf & 15.1 & -15.2 & -34.9 & 6.6 & -15.4 & -38.9 \\
\hline M158/04A ×M180/04Rf & 16.8 & -5.6 & -27.6 & 7.3 & -6.4 & -32.4 \\
\hline M158/04A ×M181/04Rf & 23.9 & 34.3 & 3.0 & 10.6 & 35.9 & -1.9 \\
\hline Standard check I ( $F_{1}$ Don 22) & 17.8 & - & -23.3 & 7.8 & - & $\begin{array}{ll}-27.8 \\
\end{array}$ \\
\hline Standard check II ( $F_{1}$ Signal) & 23.2 & 30.3 & - & 10.8 & 35.5 & - \\
\hline
\end{tabular}


As for seed and oil yields per unit area, the tested hybrids exceeded the standard ones. The hybrids Donskoy 22 and Signal released in Belarus were used as standards. Their seed oil contents were $46.69 \%$ and $49.44 \%$, respectively, under conditions of Minsk region in 2004.

The crosses M151/04A×M178/04Rf, M151/04A×M180/04Rf, M156/04A×M178/ 04Rf and M156/04A $\times$ M179/04Rf exceeded both standards in seed yield. The crosses M154/04A $\times$ M178/04Rf and M156/04A $\times$ M178/04Rf exceeded both standards in oil yield (Table 5).

\section{CONCLUSION}

The results of combining ability evaluation of the tested lines indicated that the female lines M151/04 and M156/04, having high GCA values for seed weight per head, may be used both for making high-yielding hybrids by heterosis breeding and for increasing concentration of desirable genes in the polycross. The hybrids involving these lines and the restorer M178/04Rf exceeded both standards in seed and oil yields per unit area. The obtained data indicate that possibilities exist for developing sunflower hybrids adapted to the soil and climatic conditions of the Republic of Belarus.

\section{ACKNOWLEDGEMENTS}

Help of Dr. F.I. Gorbachenko at the initial stages of the trial is gratefully acknowledged. This work was partially supported by Soya-North Co., Ltd.

\section{REFERENCES}

Silkova, T.A., Davydenko, O.G., Gorbachenko, F.I., Fomchenko, N.S., Tolstova, V.A., 2000. Pokazateli nekotoryh hozyaistvenno vazhnyh priznakov sortov i gibridov $\mathrm{F}_{1}$ podsolnechnika, vyraschennyh v usloviyah Belorussii. In: Proc. of the International scientific and practical conference dedicated to $160^{\text {th }}$ Anniversary of Belarus State Agricultural Academy, Gorky, Belarus, Jun 7-9. pp. 216-219.

Starovoitov, A.M., 2005. Gosudarstvennyi reestr sortov i drevesno-kustarnikovykh porod, Minsk, pp. 26-27.

Davydenko, O.G., Gorbachenko, F.I., Silkova, T.A., Fomchenko, N.S., Mavrishcheva, E.B., 2002. Otsenka hozyaistvenno vazhnykh priznakov u belorusskikh gibridov podsolnechnika. In: Proc. Int. Conf. on Agricultural Biotechnology, Gorki, Belarus. pp. 154-158.

Davydenko, O.G., Silkova, T.A., Gorbachenko, F.I., Fomchenko, N.S., Volotovich, A.A., 2003. Kombinatsionnaya sposobnost liniy maslichnogo podsolnechnika. Doklady NAN Belarusi. V. 47, 6:71-73.

Silkova, T.A., Davydenko, O.G., Gorbachenko, F.I., Fomchenko, N.S., Ulyanova, T.V., 2002. Vliyanie sredovykh i geneticheskikh faktorov na produktivnost gibridov $\mathrm{F}_{1}$ podsolnechnika v usloviyakh Belarusi. In: Proc. VIII BelGaS Conf., Minsk, Belarus. pp. 149-151.

Kempthorne, O., 1957. An Introduction to Genetic Statistics. John Willey and Sons, Inc., New York.

Kovacik, A., Skaloud, V., 1972. Combining ability and prediction of heterosis in sunflower (Helianthus annuus L.). Scientia Agricultural Bohemoslovaca 4(4): 263-273. 
Plokhynskiy, N.A., 1964. Nasleduemost. Novosibirsk, pp. 3-21.

Fonesca, S., Patterson, F.L., 1968. Hybrid vigour in seven parent diallel cross in common winter wheat (Triticum aestivum L.). Crop Sci. 8: 85-88.

Volotovich, A.A., Silkova, T.A., Fomchenko, N.S., Prokhorenko, O.V., Gorbachenko, F.I., Davydenko, O.G., 2005. Kombinacionnaya sposobnost i heterosis u podsolnechnika Helianthus annuus L. Vesci NAN Belarusi. Ser. bijal. navuk. 2: 47-50.

\title{
EFECTOS DE APTITUD COMBINATORIA Y EL HETEROSIS EN GIRASOL DE SELECCION BELORUSSA
}

\author{
RESUMEN
}

En combinacíon de siete líneas androestériles citoplásmicas con cuatro líneas restauradoras fueron producidos 28 hibridos sugun la esquema de testos. Los efectos de aptitud combinatoria general y específico de líneas parentales iqual que los efectos de el heterosis en hibridos $\mathrm{F}_{1}$ fueron estimados segun cuatro indicos. El rendimiento de aceite de 28 hibridos belorussos superó los ambos índices de los hibridos Donskoy 22 y Signal. El dato recibido indica la posibilidad obtener unos hibridos con perspectiva en el suelo y el clima de Belarus.

\section{EFFETS DE LA CAPACITE COMBINATOIRE ET DE L'HETEROSIS DU TOURNESOL DE LA SELECTION BIELORUSSE}

\section{RÉSUMÉ}

28 hybrides ont été obtenus conformément au schéma de tester comme résultat du croisement de sept lignées mâle stériles cytoplasmiques et quatre lignées réstauratrices. Les effets de la capacité combinatoire générale et spécifique des lignées parentales aussi bien que les effets de l'hétérosis des hybrides $\mathrm{F}_{1}$ ont été evalués sur quatre indices. Deux des vingt-huit hybrides biélorusses ont dépassé au resultat la quantité de la production de l'huile les deux standards (hybrides Donskoi 22 et Signal). Les données acquises temoignent de la possibilité de l'obtention des hybrides productifs dans les conditions de sols et de climat biélorusses. 